\title{
Attraktive Personalversicherungen
}

\section{Damit Sie und Ihre Mitarbeiter weniger Prämien zahlen}

Eine wichtige Aufgabe von FMH Insurance Services ist es, im Namen der Ärzteschaft Spezialkonditionen bei den Versicherern auszuhandeln. Als Arbeitgeber sind Sie verpflichtet, Ihr Personal zu versichern. Wir haben für Sie die zahlreichen Angebote überprüft und konnten mit der Visana einen attraktiven Rahmenvertrag aushandeln.

\section{Obligatorische Unfallversicherung (UVG)}

Seit gut zwei Jahren basieren die UVG-Prämien nicht mehr auf dem Einheitstarif des Schweizerischen Versicherungsverbandes, sondern werden von den einzelnen Gesellschaften festgelegt. Viele Versicherer haben ihre Prämiensätze bis heute nicht angepasst, weshalb sich ein Vergleich mit unserem Angebot lohnt:

\begin{tabular}{|l|l|l|}
\hline & Unsere Prämie in \% des Lohnes & Aufteilung \\
\hline Betriebsunfall & $0,088 \%$ & $100 \%$ Arbeitgeber \\
\hline Nichtbetriebsunfall & $1,132 \%$ & $100 \%$ Arbeitnehmer (in der Regel) \\
\hline
\end{tabular}

\section{Kollektive Krankentaggeldversicherung (KTG)}

Gemäss Obligationenrecht sind Sie verpflichtet, Ihren Angestellten bei Arbeitsunfähigkeit den Lohn während einer bestimmten Dauer (je nach Länge des Anstellungsverhältnisses) weiterzuzahlen. Mit einer Krankentaggeldversicherung können Sie dieses Risiko auf einen Versicherer überwälzen. Zudem profitieren Ihre Angestellten so von einer besseren Vorsorgelösung als die gesetzlichen Minimalleistungen. Auch hier bestehen teilweise grosse Unterschiede bei den Prämiensätzen der Versicherungsgesellschaften:

\begin{tabular}{|l|l|l|}
\hline Wartefrist & Unsere Prämie in \% des Lohnes & Aufteilung \\
\hline 14 Tage & $1,21 \%$ & in der Regel je $50 \%$ Arbeitgeber \\
\hline 30 Tage & $0,74 \%$ & und Arbeitnehmer \\
\hline 60 Tage & $0,43 \%$ & \\
\hline
\end{tabular}

\section{Antworttalon}

Bitte einsenden oder per Fax an: 0319595010

Vorname / Name

Adresse

PLZ / Ort

Geburtsdatum

Telefon Privat/Geschäft

Beste Zeit für einen Anruf

E-Mail-Adresse

Bitte überprüfen Sie meine Personalversicherungen und senden Sie mir eine Vergleichsofferte. (Bitte legen Sie uns eine Kopie Ihrer aktuellen Versicherungspolicen bei.)

Ich wünsche eine persönliche Beratung. Bitte rufen Sie mich an.

Ich interessiere mich für:
Steuerplanung
Pensionskasse BVG
Erwerbsunfähigkeitsvers.
Rechtsschutzversicherung
Säule $3 a$
Berufshaftpflichtversicherung
O

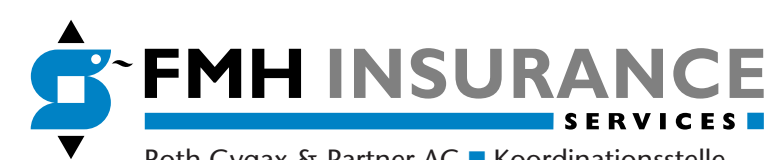

Roth Gygax \& Partner AG $\square$ Koordinationsstelle Moosstrasse 2 a 3073 Gümligen

Telefon 0319595000 口 Fax 0319595010 